\title{
Sociétés, monnaie et politique : éléments pour une théorie de l'institution monétaire et une typologie des formes historiques de sa régulation politique
}

\section{Éric Pineault}

\section{Numéro 32, 1999}

La politique en otage

URI : https://id.erudit.org/iderudit/1002399ar

DOI : https://doi.org/10.7202/1002399ar

\section{Aller au sommaire du numéro}

\section{Éditeur(s)}

Département de sociologie - Université du Québec à Montréal

ISSN

0831-1048 (imprimé)

1923-5771 (numérique)

Découvrir la revue

Citer cet article

Pineault, É. (1999). Sociétés, monnaie et politique : éléments pour une théorie de l'institution monétaire et une typologie des formes historiques de sa régulation politique. Cahiers de recherche sociologique, (32), 47-83.

https://doi.org/10.7202/1002399ar

\section{Résumé de l'article}

Cet article est une contribution au développement d'une théorie institutionnaliste de la monnaie et de la régulation monétaire. Il vise à éclairer le sens des mutations contemporaines des pratiques et politiques monétaires qui sont au coeur du processus de mondialisation du capitalisme, en explorant les racines historiques de la régulation et de l'institution monétaires. Dans une première partie, de nature épistémologique, l'auteur s'emploie, en s'inspirant des travaux de Michel Freitag, à la construction d'une théorie de l'institution monétaire. Cette démarche lui permet de proposer une phénoménologie des pratiques et de la régulation monétaires dans les sociétés traditionnelles et modernes et d'esquisser une typologie des formes de la régulation monétaire. Cette typologie s'inscrit dans la perspective de l'analyse des formes de l'État et de la régulation politique de Bourque et Duchastel. L'article se termine par un examen plus précis de la régulation monétaire néolibérale et laisse entendre que celle-ci participe directement à la légitimation et à la production de l'infrastructure institutionnelle et organisationnelle nécessaire aux mutations actuelles de la monnaie et à l'absorption de l'institution monétaire dans la sphère de la finance privée. 


\section{Sociétés, monnaie et politique: éléments pour une théorie de l'institution monétaire et une typologie des formes historiques de sa régulation politique*}

Les transformations actuelles du capitalisme, comprises comme la «mondialisation de l'économie», s'accompagnent d'une mutation fondamentale de la nature et de la fonction sociale de la monnaie, ainsi que d'une recomposition des rapports entre État et économie, qui se manifeste avec le plus d'acuité dans les domaines financier et monétaire'. Paradoxalement, pendant que les expressions typiques de la valeur marchande d'une monnaie nationale, taux de change et taux d'inflation, sont devenues les variables déterminantes de l'évaluation de la rentabilité de toute opération économique «globale» par les acteurs économiques dominants (les multinationales), la régulation monétaire échappe à l'État en se dépolitisant. La régulation monétaire contemporaine, néolibérale, se présente sous la forme d'une autonomisation de la politique monétaire et des banques centrales par rapport aux pouvoirs législatif et exécutif ainsi que par rapport aux autres instances politiques de régulation économique. Et les divers projets de monnaie unique, de l'euro à l'éventuelle monnaie nord-américaine ${ }^{2}$, proposés en réaction à ces transformations vont tous

Cet article reprend une partie de mon mémoire de maîtrise, soit les chapitres 2 , «Repères épistémologiques pour une sociologie de l'économie», et 3, "Ébauche d'une théorie de 1'institution et de la régulation monétaire» (É. Pineault, La Banque du Canada et la dissolution de l'État-providence canadien, éléments pour une sociologie de la régulation monétaire, département de sociologie, Université du Québec à Montréal, 1998). Je tiens à remercier le FCAR de son soutien financier pour la rédaction de ce mémoire.

H. Bourguinat, Finance internationale, Paris, PUF, 1995.

2 D'ailleurs, au Québec, ce projet se présente sous la forme d'une version «post-sociale- 
dans le même sens, soit celui d'un approfondissement et d'une consolidation de la régulation monétaire néolibérale et donc de la dépolitisation du rapport de la société à la monnaie.

Ces transformations dans le domaine de l'économie politique accompagnent une mutation de la forme même de la monnaie ainsi que de la nature et de l'extension des pratiques qu'elle médiatise ${ }^{3}$. La monnaie, qui médiatise, oriente et organise de plus en plus de pratiques sociales, ne se présente plus sous la forme du symbole, billet de la banque centrale ou pièce frappée, portant la marque de la souveraineté politique, et donc transposant et imposant la présence et la sanction du politique au cœur des rapports privés. Une des caractéristiques centrales de l'institution monétaire était justement cette capacité d'insérer symboliquement et juridiquement les transactions économiques privées dans l'espace public intermédiaire, soit l'espace entre le politique et l'intimité que constituait l'espace civil. La multiplication des nouvelles formes monétaires électroniques et communicationnelles, que celles-ci se déploient au niveau macro sur les marchés monétaires mondialisés ou au niveau micro au cours des transactions quotidiennes, a pour effet de «reprivatiser» l'espace des pratiques économiques, de dissoudre l'espace civil dans des circuits privatisés, en plus de favoriser l'absorption de la sphère monétaire par la sphère de la finance privée 4 . Cette absorption n'est pas balisée par une structure institutionnelle qui médiatiserait ces deux ordres de rapports économiques et, donc, qui conférerait aux pratiques monétaires une autonomie relative au regard de la finance. L'espace des transactions et des rapports orientés par la monnaie devient de plus en plus financiarisé par l'indifférenciation des moyens de paiement et des moyens de crédit à l'intérieur des circuits et réseaux monétaro-financiers privatisés ${ }^{5}$. Ce qui peut être compris comme une transformation de la régulation monétaire correspond ainsi à la fois à une transformation du rapport du politique avec la sphère des pratiques économiques et à une transformation de la forme

démocrate» de la «piasse à Lévesque» des souverainistes.

3 Voir, à ce sujet, J. Kurtzmann, The Death of Money, New York, Back Bay Books, 1993, et B. Perret, Les nouvelles frontières de l'argent, Paris, Seuil, 1998.

4 La création des banques centrales et la mise en place de la régulation monétaire moderne impliquaient déjà l'absorption de la sphère des pratiques monétaires par la finance publique. Comme je le montrerai plus loin, cette absorption s'est faite de l'extérieur de la sphère économique par l'assujettissement politique de la monnaie par la finance d'État.

5 A. J. Haesler, Sociologie de l'argent et postmodernité, Genève, Droz, 1994. 
même de la monnaie à titre de médiation économique.

Ces phénomènes retiennent l'attention de plus en plus de spécialistes en sciences sociales, ils ont été vus par plusieurs analystes, notamment Bernard Perret ${ }^{6}$, Michel Aglietta ${ }^{7}$ et André Orléan ${ }^{8}$, comme une consolidation d'un «pouvoir de l'argent» (la sphère des pratiques monétaires) sur les sociétés et les économies contemporaines. Ces auteurs ont tenté d'aller au-delà d'une simple description des ravages sociaux de la monnaie devenue marchandise ${ }^{9}$, en formulant une critique du fétichisme de la monnaie-marchandise par le biais d'une analyse de l'infrastructure institutionnelle qui a permis sa construction. En élaborant une théorie «qualitative» de la monnaie qui s'inspire plus de la sociologie et de l'anthropologie que de la science économique néoclassique, ces auteurs ont osé contester le monopole de la science économique et de sa branche la plus ésotérique, la théorie monétaire et financière, dans la définition et l'analyse de la monnaie. Ils ont pour la première fois opposé une théorie institutionnelle de la monnaie à la théorie classique de la monnaie, propre à la science économique.

Le présent article se veut une contribution à ce travail. Sa visée précise est de comprendre les racines historiques de la régulation monétaire et de l'institution monétaire afin de mieux éclairer l'importance et le sens des mutations contemporaines que j'ai évoquées plus haut. La plus grande partie de ce texte sera donc consacrée à la construction d'une théorie de l'institution monétaire et des modalités de régulation politique des pratiques qu'elle régit dans les sociétés traditionnelles et modernes. Cette théorie s'inspire de la théorie de l'institution et du politique développée par Michel Freitag ainsi que des travaux de Jacques Beauchemin, Gilles Bourque et Jules Duchastel sur la régulation politique et les formes de l'État ${ }^{10}$. Cet ancrage sociologique me permettra de proposer une lecture des

6 B. Perret, ouvr. cité.

7 M. Aglietta et J. Cartelier, «L'ordre monétaire des économies de marchés», dans M. Aglietta et A. Orléan (dir.), La monnaie souveraine, Paris, Odile Jacob, 1998. p. 129-157.

8 A. Orléan, «La monnaie auto-référentielle: réflexions sur les évolutions monétaires contemporaines», dans M. Aglietta et A. Orléan (dir.), ouvr. cité, p. 359-386.

${ }^{9}$ Ce à quoi se limite l'analyse de J. Kurtzman (ouvr. cité).

11) Voir, à ce sujet: J. Beauchemin, G. Bourque et J. Duchastel, «Du providentialisme au néolibéralisme: de Marsh à Axworthy. Un nouveau discours de légitimation de la régulation 
travaux actuels sur l'institution et la régulation monétaires qui prendra la forme d'une phénoménologie de l'institution monétaire et d'une typologie des formes historiques du rapport entre le politique et la monnaie.

Le point de départ obligé d'une telle démarche théorique et historique est un regard critique sur l'analyse de la monnaie que font l'économie politique moderne et la science économique «orthodoxe» contemporaine. L'objectif de ce survol ${ }^{11}$ est de dégager les acquis et les limites des approches «conventionnalistes» de la monnaie et d'exposer la compréhension de la monnaie, de ses fonctions et de son rapport avec le politique qui orientent actuellement les politiques monétaires de la majorité des banques centrales. Une épistémologie de la notion d'institution en sociologie économique et politique sera l'étape intermédiaire entre cette critique et l'analyse phénoménologique de la monnaie et la typologie des modalités historiques de la régulation monétaire. Cette typologie se terminera par une présentation succincte des postulats du monétarisme en tant que représentation idéologique qui structure les régulations monétaires néolibérales. Nous verrons comment celle-ci contribue à la légitimation et donc à la production de l'infrastructure institutionnelle et organisationnelle nécessaire aux mutations actuelles de la forme de la monnaie et à l'absorption de cette sphère de pratique par la finance privée.

\section{La conception économique de la monnaie dans la modernité: de la fable du troc à la théorie quantitative de la monnaie, la consolidation du système capitaliste et la régulation économique libérale}

Dans le discours de l'économie politique moderne, la monnaie est comprise avant tout comme un «instrument» qui se définit en vertu des «fonctions» qu'il remplit dlans un système d'échange, cette forme de rapport social qu'est l'échange «marchand» étant considérée comme naturelle et universelle. Pour l'économie politique moderne, et la science économique en général, la monnaie est ainsi une «convention» sur laquelle

sociale», Cahier de recherche sociologique, no 24, 1995, p. 15-47.

1 Survol qui sera d'autant plus rapide que la critique des limites de ces théories a été maintes fois effectuée, en commençant par Marx dans sa Contribution à la critique de l'économie politique en 1857. Pour une critique contemporaine, voir, entre autres, M. Aglietta et A. Orléan, La violence de la monnaie, Paris, PUF, 1982. 
s'entendent les participants à l'échange, parce qu'elle facilite leurs interactions, plutôt qu'une institution qui institue une forme déterminée de rapport social. La différence entre convention et institution est fondamentale au regard du développement qui va suivre. Les théoriciens de la monnaie-convention postulent que la monnaie apparaît à l'intérieur de la dimension horizontale des pratiques sociales de "premier degré» d'acteur à acteur - et qu'elle se moule ainsi à une forme de rapports (dans ce cas, les rapports marchands) qui lui préexiste. Cela explique d'ailleurs pourquoi la monnaie est, dans le discours de l'économie politique moderne, «monnaie-marchandise», c'est-à-dire que sa forme apriorique est celle de la marchandise et que sa nature d'institution politique est considérée comme une caractéristique secondaire. Une théorie de la monnaie-institution tient compte de la dimension verticale de la production politique d'une forme de rapport social qui fonde l'espace économique par le biais de la création de l'institution monétaire et de la régulation que celle-ci appelle.

Le point de départ «classique» de l'analyse de la monnaie-convention est la «fable du troc ${ }^{12} »$, une expression de l'historien de l'économie JeanMichel Servet ${ }^{13}$ qui montre que le troc est un des mythes fondateurs d'une science de l'économie en tant que la notion fait abstraction de toutes les autres formes de rapports sociaux. La fable se présente comme suit: deux producteurs en situation d'interdépendance se retrouvent dans l'impossibilité de répondre, voire de les anticiper, aux besoins de l'autre; or cette anticipation est une condition préalable de l'échange qui prend la forme du troc (par exemple, une poche de pommes de terre contre une veste de laine). La monnaie, agissant à titre de réserve de pouvoir d'achat, c'est-àdire comme matérialisation de pure valeur d'échange, est ainsi inventée de façon consensuelle par les échangistes pour solutionner le problème de la «double cö̈ncidence des besoins ${ }^{14} »$. Cette fable suppose l'existence d'un

12 Pour une présentation paradigmatique de la fable du troc dans sa version classique, voir A. Smith, «Of the origin and use of money», chap. IV, dans The Wealth of Nations, livre 1, Londres, Penguin, 1986 (1776) p. 126-150; pour une présentation «contemporaine», je renvoie au chapitre introductif sur la monnaie de n'importe quel ouvrage de science économique. À titre d'exemple: P.-J. Lehmann, Économie monétaire, Paris, Seuil, 1997 , p. 4-5.

13 J.-M. Servet, «La monnaie contre l'État ou la fable du troc», dans P. Kahn (dir.), Droit et monnaie. États et espace monétaire transnational, Dijon, Litec, 1988.

${ }^{14}$ Cette formule est celle qui est typiquement employée dans les manuels d'économie. 
espace d'interaction sociale vidé de tout contenu normatif a priori, le marché abstrait, et son fonctionnement implique à terme la création d'un médium «structurant» les échanges dans cet espace selon un axe temporel (thésaurisation), spatial (moyen de paiement obligatoire) et matériel (unité de référence). De cette déduction théorique du «besoin» de la monnaie, en tant que médiation formelle des intentions à l'intérieur du marché, l'analyse économique peut inférer les fonctions de la monnaie en tant qu'instrument d'échange universel. Ces fonctions théoriques sont au nombre de trois: la monnaie comme unité de compte, comme moyen de paiement légal et comme instrument de thésaurisation. Elles font l'objet d'une description plus complète dans le tableau 1.

La science économique a construit une succession de théories de l'autorégulation monétaire en explorant les relations formelles entre ces fonctions qu'elle a transformées en variables mathématiques, soit: $P$ (prix: un indice du niveau des prix des objets offerts ou en vente dans un marché donné), $M$ (masse monétaire: la somme de l'ensemble des unités monétaires dans un marché donné) et $\mathrm{V}$ (vitesse: la vitesse de circulation des unités monétaires dans un marché donné). Dans son expression la plus simple, la relation entre les trois fonctions de la monnaie se résume dans l'équation $\mathrm{P}=\mathrm{M} \times 1 / \mathrm{V}$, c'est-à-dire que la totalité des prix est fonction de la masse monétaire et de sa vitesse de circulation. C'est là la base de la théorie quantitative de la monnaie. La totalité des prix n'est en fait que la totalité des «unités de compte» nécessaires à l'achat de la totalité des objets disponibles; ce rapport exprime donc la «valeur de la monnaie». Pour l'économie politique et la science économique qui lui succède, la monnaie se réduit à une convention sociale permettant l'autorégulation des pratiques économiques.

Voir, par exemple: R. A. Shearer et autres, Economics of the Canadian Financial System. Theory, Policy and Institutions, 5e éd., Toronto, Prentice-Hall, 1995, chap. 1 et 2, surtout p. 5-7; G. F. Boreham et autres, Money Banking and Finance, 4e éd., Toronto, Holt, Rhinehart and Winston, 1993, chap. 1 p. 6-13; G. Rabior, Money and Monetary Policy in Canada, Toronto, Canadian Foundation for Economic Education, 1994, p. 2-10. 


\section{Tableau 1}

\section{Les fonctions de la monnaie-convention}

\begin{tabular}{|c|c|c|c|}
\hline Fonction & Unité de compte & $\begin{array}{l}\text { Moyen de paiement } \\
\text { légal }\end{array}$ & $\begin{array}{l}\text { Instrument de } \\
\text { thésaurisation }\end{array}$ \\
\hline Nature & $\begin{array}{l}\text { La monnaie est } \\
\text { l'instrument universel } \\
\text { de mesure de la } \\
\text { valeur d'échange; } \\
\text { elle permet la } \\
\text { traduction des } \\
\text { qualités (valeur } \\
\text { d'usage) en quantités } \\
\text { (valeur d'échange) à } \\
\text { l'intérieur d'un } \\
\text { système d'évaluation. }\end{array}$ & $\begin{array}{l}\text { La monnaie a une } \\
\text { capacité libératoire } \\
\text { universelle. } \\
\text { L'échange d'une } \\
\text { quantité déterminée } \\
\text { de monnaie éteint } \\
\text { obligatoirement une } \\
\text { dette correspondante; } \\
\text { la monnaie est donc } \\
\text { un intermédiaire } \\
\text { «irrécusable» des } \\
\text { échanges } \\
\text { économiques. }\end{array}$ & $\begin{array}{l}\text { La monnaie est une } \\
\text { réserve de pouvoir } \\
\text { d'achat; en tant que } \\
\text { médiation formelle } \\
\text { exprimant la pure } \\
\text { quantité, elle doit } \\
\text { conserver dans le } \\
\text { temps sa fonction } \\
\text { libératoire et d'unité } \\
\text { de compte. Elle } \\
\text { permet de différer le } \\
\text { pouvoir d'achat } \\
\text { acquis lors d'un } \\
\text { échange. La monnaie } \\
\text { étant pure valeur } \\
\text { d'échange, elle n'a } \\
\text { pas besoin de subir } \\
\text { une transformation } \\
\text { pour passer de la } \\
\text { qualité à la quantité. }\end{array}$ \\
\hline Conséquence & $\begin{array}{l}\text { La monnaie dissout la } \\
\text { qualité des choses } \\
\text { dans une expression } \\
\text { quantifiée; le rapport } \\
\text { entre la monnaie et } \\
\text { les choses se présente } \\
\text { sous la forme du prix }\end{array}$ & $\begin{array}{l}\text { Cela suppose qu'il y } \\
\text { ait une équivalence } \\
\text { stricte entre les } \\
\text { transactions } \\
\text { économiques et la } \\
\text { vitesse de circulation } \\
\text { de la monnaie, } \\
\text { puisque chaque } \\
\text { transaction de } \\
\text { marchandise } \\
\text { implique en contre- } \\
\text { partie un échange } \\
\text { monétaire. }\end{array}$ & $\begin{array}{l}\text { Cela implique qu'il y } \\
\text { ait une relation stable } \\
\text { et formelle entre les } \\
\text { prix et la monnaie; la } \\
\text { monnaie a une valeur } \\
\text { déterminée. }\end{array}$ \\
\hline $\begin{array}{l}\text { Manifestation } \\
\text { économique }\end{array}$ & Prix-P & $\begin{array}{l}\text { Vitesse de } \\
\text { circulation- V }\end{array}$ & Masse monétaire- $\mathrm{M}$ \\
\hline
\end{tabular}

En fait, cette façon d'appréhender les mécanismes monétaires renvoie à l'extension concrète du marché et, donc, de la monétarisation croissante des pratiques sociales dans les sociétés occidentales au début de la 
modernité. Les premières formulations de la théorie ${ }^{15}$ de l'autorégulation monétaire sont contemporaines de la totalisation des pratiques économiques en système institutionnel pendant le capitalisme mercantile. Les variables de l'équation monétaire «quantitativiste» peuvent être mises en relation formelle (économique) les unes avec les autres à cette époque justement parce que chacune des fonctions de la monnaie est libérée de l'emprise d'une multiplicité hétérogène de régulations d'ordre politique et culturel propres aux sociétés traditionnelles. Ainsi, la liberté des prix ( $\mathrm{P}$ ) implique la fin de l'idéal aristotélicien du «juste prix» et du droit de régulation des prix par les autorités municipales ou par les corporations de métier; la libération de la circulation monétaire (V) a signifié la fin du droit des princes locaux d'obliger la refonte des monnaies provenant d'une autre région ainsi que l'unification dans le temps et l'espace des marchés régionaux et locaux en un «marché national»; l'autonomisation de la fonction de thésaurisation a pris la forme de la libération du crédit (M) et a signifié la fin de l'interdiction de l'usure et la reconnaissance du droit de fixer arbitrairement (économiquement) les taux d'intérêt. On pourrait avancer que le discours sur la monnaie de l'économie politique moderne légitime cette autonomisation et cette totalisation sur soi des fonctions monétaires autant qu'il les explique. La régulation monétaire «moderne» qui se nourrit de ce discours participe ainsi à l'unification des pratiques économiques en système, en faisant de l'autonomie formelle et de la clôture opérationnelle de ces pratiques un a priori.

Ce survol nous permet de constater les limites d'une théorie purement conventionnelle de la monnaie. Au fond, cette théorie n'explique pas la nature sociale de la monnaie, elle participe de la légitimation d'un mode particulier de régulation monétaire et, par conséquent, de construction d'une représentation sociale - idéologique - du rapport politique à la monnaie. En effet, la théorie de l'autorégulation monétaire sert à légitimer la mise en place d'une régulation politique de l'institution monétaire de type libéral, tout comme sa reformulation monétariste servira à la légitimation de la transition de la régulation monétaire providentialiste à une régulation néolibérale. La construction de cette théorie de l'autorégulation monétaire nécessite le recours à la fable du troc à titre de discours fondateur de la monnaie-convention pour s'opposer à l'évidence

15 Par exemple chez des penseurs préclassiques tels que Jean Bodin, John Locke et David Hume, pour ne nommer qu'eux. 
empirique de la préexistence de la monnaie-institution au capitalisme moderne et des idéologies légitimant les modes de régulation traditionnels de la monnaie. Les théories de la monnaie-convention n'expliquent pas la monnaie, elles produisent une représentation sociale de la monnaie particulière à un contexte historique et propice au projet politique d'une classe sociale, la bourgeoisie. Évidemment, il nous faut tenir compte de cette représentation particulière de la monnaie, et un regard sociologique sur la monnaie ne peut pas l'ignorer sous prétexte qu'il s'agit d'un pur phénomène idéologique qui voilerait le réel. Nous devons construire une théorie et une histoire sociale de la monnaie capables d'incorporer cette représentation particulière ainsi que celles qui l'ont précédée et suivie, et de leur donner un sens. La première étape de cette démarche prend la forme d'un questionnement épistémologique sur la nature sociologique des médiations formelles des pratiques économiques, telles que la monnaie.

\section{Quelques présupposés épistémologiques concernant la notion d'institution en sociologie de l'économie}

Du point de vue d'une sociologie de l'économie, l'activité économique n'est pas une dimension naturelle de l'activité humaine et de la vie sociale, comme le voudraient les théories de l'autorégulation monétaire, mais plutôt une forme particulière de rapport social existant historiquement dans certaines sociétés. Les «pratiques économiques» se reproduisent, comme toute pratique sociale, par le biais de médiations normatives qui contribuent à la reproduction non seulement de cette sphère de la pratique plus ou moins autonomisée qu'est l'économie, mais aussi de la société ${ }^{16}$.

\footnotetext{
${ }^{16}$ Cette perspective nous éloigne donc de la conception néoclassique de l'économie ainsi que des conceptions néomarxistes d'inspiration structuraliste. Elle s'apparente cependant à celle des institutionnalistes qui considèrent que l'activité économique se reproduit par le biais d'un ensemble de médiations institutionnelles et que l'économie elle-même apparaît dans la société en tant qu'institution. Je dois préciser que la notion d'institution telle que je l'emploie dans mon analyse est sociologique plutôt qu'économique. Je ne peux adhérer au postulat sous-jacent à certaines analyses institutionnalistes d'une émergence naturelle ou spontanée des «institutions» économiques à l'intérieur des sociétés comme structure de régulation d'une «dimension» inhérente à la vie sociale. En postulant le caractère naturel des institutions économiques, les institutionnalistes n'évacuent pas les conflits sociaux au sujet de la nature de ces institutions; ce qui est «naturalisé»», c'est la nécessité des rapports économiques, non pas leur configuration particulière qui, elle, serait contingente. Je ne puis davantage souscrire à la conception de rapports économiques des «conventionnalistes» comme rapports contractuels et marchands (régulation de l'échange) ou, plus subtilement,
} 
L'institution n'est, sociologiquement, ni une "convention" sur laquelle s'entendraient un ensemble d'acteurs afin de structurer plus rationnellement leurs interactions ni la manifestation d'une structure de production sous-jacente aux activités observables, mais bien une catégorie de pratiques définie, légitimée et sanctionnée par une superstructure politique. L'institution ne renvoie pas seulement à une forme particulière de médiation des pratiques sociales qui pourrait analytiquement être différenciée des autres formes par sa permanence, son universalité et son caractère conventionnel (chez Marcel Mauss, par exemple), elle renvoie à une forme de médiation propre à un type historique de société et ses caractéristiques sont celles-là mêmes d'un mode de reproduction spécifique de la société. D'un point de vue sociologique, les institutions économiques «n'émergent pas»; à titre d'institutions, elles sont produites et imposées à la société par le politique en tant que normes formelles des pratiques.

Cette définition de l'institution comme médiation formelle de l'activité sociale caractéristique des sociétés dont le procès de reproduction est avant tout politique a été développée par Michel Freitag dans le deuxième tome de son ouvrage Dialectique et société ${ }^{17}$. Le concept d'institution renvoie à la forme nécessaire des médiations de l'activité sociale plutôt qu'au contenu particulier de ces médiations. Dans son sens le plus général, on peut dire que le concept d'institution renvoie à la présence d'activités sociales qui, plutôt que d'être «régies ou orientées par des normes intériorisées par les acteurs, [sont le produit] d'une activité sociale de second degré qui établit ou définit les règles qui vont s'imposer de l'extérieur et par anticipation aux acteurs sociaux dans l'accomplissement de leurs pratiques de premier degré, et qui dispose également de la capacité

par les «polanyiens», comme rapports de production répondant à des «besoins» (régulation de la production et de la circulation). Voir, par exemple, les travaux d'O. Williamson, The Economic Institutions of Capitalism, New York, Free Press, 1985, de M. Granovetter, notamment "Economic institutions as social constructions: A framework for analysis», Acta Sociologica, vol. 35, 1992, p. 3-12, ainsi que les travaux des fondateurs, entre autres $\mathrm{K}$. Polanyi, «L'économie en tant que procès institutionnalisé», dans $\mathrm{K}$. Polanyi et $\mathrm{C}$. Arensberg (dir.). Les systèmes économiques dans l'histoire et dans la théorie, Paris, Larousse, 1974, p. 239-260.

17 M. Freitag, Dialectique et société: culture, pouvoir, contrôle, les modes de reproduction formels de la société, t. II: Le mode de régulation politico-institutionnel et le développement de la praxis historique, Montréal et Genève, Éditions Saint-Martin et L’Âge d'homme, 1986, p. 161-311. 
de les sanctionner soit, a priori, en déterminant la valeur sociale qui leur est attachée, soit après coup, en intervenant pour en modifier les résultats ou les conséquences ${ }^{18} »$. Freitag précise ailleurs que «l'institution ne concerne donc plus telle ou telle action concrète et particulière, mais des catégories générales et abstraites d'actions ${ }^{19}{ }^{\prime}$.

L'institution en tant que forme est la synthèse d'une médiation de la pratique, médiation qui se décompose en trois dimensions structurelles, lesquelles renvoient d'ailleurs au moment du procès d'institutionnalisation politique: 1) la production d'une définition qui objective la pratique en une catégorie abstraite; 2) la capacité de sanction conditionnelle afin d'actualiser cette définition dans la sphère de la pratique; et 3) une pratique de légitimation idéologique de cette production normative qui l'inscrit dans la hiérarchie des régulations sociétales et qui, par là même, justifie cette norme ou ce champ normatif comme moment de reproduction de la société et confirme parallèlement la légitimité de l'activité superstructurelle de production normative et de sanction des pratiques. Ce procès implique donc la matérialisation d'un axe vertical en position d'extériorité par rapport à des activités de base comme «superstructure institutionnelle» dont la forme typique est politique ${ }^{20}$.

Une institution économique telle que la monnaie n'émerge pas spontanément à la suite d'une sélection des formes de régulation les plus efficientes dans la logique qu'elle institue ${ }^{21}$; elle est le produit d'un procès d'institutionnalisation politique qui transcende et domine cette logique, une domination qui correspond aux trois dimensions de la médiation institutionnelle dégagées ci-dessus. Ce n'est que dans un deuxième temps que cette sphère de pratique institutionnelle, ainsi légitimée, peut s'affranchir de cette domination immédiate par le politique en y interposant son propre échafaudage institutionnel. L'émancipation relative des sphères institutionnelles de la pratique sociale par rapport à la domination politique n'est possible que dans la deuxième phase de l'institutionnalisation, qui est propre aux sociétés modernes.

\footnotetext{
18 M. Freitag, «La métamorphose», Société, nos 12-13, 1995, p. 55.

19 M. Freitag, ouvr. cité. p. 217.

20) Ibid., p. 218.

21 Comme le voudrait le raisonnement tautologique de la fable du troc.
} 
Pour conclure cette analyse de la notion d'institution, examinons rapidement les formes historiques qu'a prises le mode de reproduction politico-institutionnel de la société. Freitag distingue deux cycles dans ce mode de reproduction. Le premier caractérise les sociétés traditionnelles à l'intérieur desquelles le pouvoir politique s'est constitué en instance de reconnaissance et d'arbitrage des puissances d'agir et des formes de pratiques individuelles au nom du maintien de l'unité de la société compris comme maintien d'un ordre transcendant extra-mondain voulu par une «personne» divine. C'est au nom de cette volonté divine que le pouvoir sanctionne les pratiques particulières (par les juges), ce qui a pour effet, mais seulement a posteriori, d'abstraire de celle-ci des «définitions» institutionnelles légitimes. Le deuxième cycle du mode de reproduction politico-institutionnel se rattache aux sociétés «modernes» à l'intérieur desquelles cette capacité d'institutionnalisation a elle-même été «institutionnalisée». Les sociétés modernes sont donc caractérisées par le déploiement d'une pratique de troisième degré de «régulation formelle des médiations formelles», c'est-à-dire une pratique de régulation institutionnelle des institutions. Cette pratique de troisième degré n'est dans le fond que l'emprise réflexive de la société sur la superstructure institutionnelle qui la régit. Il en résulte non seulement l'émergence d'un nombre indéfini de niveaux de régulation, mais aussi la constitution de champs de pratiques institutionnels, ou de sous-systèmes institutionnels, plus ou moins autonomes selon le degré de développement de leurs propres niveaux de régulation.

Une grande partie de ces institutions - le marché, le contrat, la monnaie, la propriété, etc. - sont nées dans des sociétés où la logique de reproduction correspondait au premier cycle d'institutionnalisation, et ce n'est que dans le passage au deuxième cycle, c'est-à-dire avec l'avènement de la modernité, que ces médiations institutionnelles ont pu se totaliser en «système économique» comprenant en lui-même sa propre cohérence et son autonomie relative par rapport au reste de la société. Cette autonomisation et cette systématisation n'auraient pas été possibles sans le travail idéologique de l' «économie politique» comme discours de légitimation de l'institutionnalisation de cette sphère de pratiques à partir d'un ensemble de pratiques institutionnelles disparates. On comprend maintenant mieux la fonction fondatrice de la fable du troc. Cette unification systématique de laquelle émerge l'économie dans les sociétés modernes implique à la fois une hiérarchisation des médiations 
institutionnelles à l'intérieur du système économique comme tel et le déploiement d'un ensemble hiérarchisé de régulations politiques surplombant ce système.

C'est à ce «modèle» que nous allons nous référer pour comprendre l'émergence de l'institution monétaire dans les sociétés occidentales prémodernes et, par la suite, des institutions politiques de régulation monétaire, c'est-à-dire les banques centrales, dans les sociétés modernes. L'émergence de la régulation monétaire, en tant que pratique de «troisième degré» de régulation des pratiques d'une institution économique, sera d'ailleurs comprise comme moment participant à l'institutionnalisation en général de l'économie comme système social et en particulier des systèmes financiers à titre de dimension interne de ce système.

\section{Une phénoménologie de l'institution monétaire}

Par le biais de la fable du troc, le discours de l'économie politique réussit à éviter d'avoir à faire une analyse empirique de l'émergence de la monnaie dans les sociétés traditionnelles, préférant une représentation idéalisée qui suppose la naturalité des pratiques économiques. C'est cette question que je veux maintenant explorer afin de dégager les grandes lignes du procès d'institutionnalisation politique de l'institution monétaire. Pour ce faire, je m'appuierai sur des synthèses des recherches archéologiques et historiques les plus récentes sur l'invention de la monnaie en Asie Mineure, produites par les historiens Jean-Marie Thiveaud et Sylvain Piron ${ }^{22}$, ainsi que par Michel Aglietta et André Orléan ${ }^{23}$. L'analyse de l'émergence de l'institution monétaire commencera donc par une herméneutique de la naissance du monnayage dans l'Antiquité. Je crois que c'est dans la forme même de la monnaie que réside le secret de son caractère institutionnel. De plus, l'analyse de ce caractère institutionnel doit pouvoir intégrer de façon cohérente les acquis de ma critique des théories "conventionnalistes» de la monnaie, soit la triple dimension de son travail de médiation des pratiques sociales tel que réifié dans la notion des fonctions monétaires: unité de compte, moyen de

22 J.-M. Thiveaud et S. Piron, «De la monnaie électronique à l'invention de la monnaie d'électron», Revue d'économie financière, no 32, 1995, p. $271-293$.

23 M. Aglietta et A. Orléan, La violence de la monnaie, ouvr. cité. 
paiement, réserve de valeur. En fait, l'hypothèse est que ces fonctions pseudo-naturelles de la monnaie sont véritablement des catégories nouvelles de pratiques sociales légitimées par le pouvoir politique qui se trouvent représentées dans la forme même la monnaie.

Pile et face: la naissance de la monnaie dans les sociétés traditionnelles de l'Antiquité

Bien que des métaux précieux, sous la forme de lingots, et certaines céréales aient été utilisés comme instruments d'échange ou unités de compte dans plusieurs anciennes civilisations, les premières pièces de monnaie sont apparues quasi simultanément au vile siècle av. J.-C. en Asie Mineure, en Inde et en Chine. Il semblerait que la première émission de monnaie eut lieu au royaume de Lydie entre 630 et 600 av. J.-C. Lieu de passage des caravanes provenant de l'Asie en route vers les cités grecques, la Lydie constituait un intermédiaire culturel et politique important entre les civilisations hellénique et mésopotamienne ainsi qu'une puissance régionale qui régna sur les cités ioniennes alors que celles-ci connaissaient leur apogée culturelle. Deux expressions populaires rappellent le lien entre la Lydie et la monnaie: «c'est un vrai pactole» et «riche comme Crésus». Pactole est le nom d'un fleuve coulant non loin de la capitale du royaume dont les coulées alluviales fournissaient aux rois lydiens l'or et l'argent leur permettant de fabriquer la monnaie, et Crésus est le nom d'un des derniers rois ayant régné sur la Lydie avant la conquête perse.

Selon Thiveaud et Piron, la «monnaie» lydienne était fort probablement à l'origine un «talisman» sacré dont la fonction était religieuse et dont la fabrication était monopolisée par le pouvoir royal. Le talisman qui deviendra monnaie portait déjà en lui le pouvoir de faire circuler dettes et créances, mais celles-ci n'existaient pas tant directement entre les hommes qu'entre le monde divin et le monde terrestre et étaient médiatisées par la personne royale. La transformation des talismans en monnaie se produira parallèlement à la sécularisation du pouvoir de la royauté lydienne.

Les pièces de monnaie émises par les rois lydiens se différencient des lingots de métal utilisés à l'époque en Mésopotamie par leur taille, par leur composition métallique (alliage plutôt que métal pur) et, surtout, par la présence du sceau royal frappé sur une des faces et une série de marques de 
poinçon au revers indiquant la position de la pièce dans un système pondéral d'origine babylonienne. «Les deux traits singuliers de l'invention technologique des pièces de monnaie métallique, en Lydie, au vile siècle avant J.-C., se résument donc par l'apposition de l'empreinte d'un sceau et la division de la masse métallique en éléments proportionnés. ${ }^{24}{ }{ } \mathrm{La}$ monnaie lydienne comportait ainsi déjà la dualité pile et face des monnaies modernes. Le côté «face» se rapporte, par le sceau royal, à la puissance de la souveraineté politique : «Lorsque les premières monnaies sont munies d'un sceau, les attributs surnaturels, divins, ésotériques, associés à la personne royale sont ainsi transmis à la pièce de métal qui en reçoit l'empreinte. Et le métal lui-même participe du sacré puisqu'il provient directement du trésor royal et bénéficie déjà de ces vertus mystérieuses. ${ }^{25}$ " Et le côté «pile» se rapporte à la relation d'équivalence formelle instituée entre les pièces, transformant le rapport qualitatif à la puissance souveraine en un rapport quantitatif, fractionnable, échangeable et accumulable:

Les pièces de monnaie ouvrent un espace de calcul qui distingue l'être ou la chose de son poids. Cette ouverture permet de comparer les choses entre elles, en remplaçant la conversion pondérale par une autre forme de conversion qui, au nom de la gloire du roi manifestée dans son sceau, introduit dans le monde des êtres et des choses l'ordre de la valeur. Car la valeur, telle que les linguistes la définissent dans le monde archaïque, s'applique jusque-là à des cas très limités. Elle est d'abord la valeur personnelle d'un homme ${ }^{26}$, le mérite moral attaché à son courage mais aussi le prix qu'il faudra donner pour le racheter de sa servitude, la libération passant par l'acte primordial du rachat ${ }^{27}$.

On voit ici les racines de deux des trois fonctions de la monnaie définies dans la section précédente, on les découvre en plus sans faire intervenir l'existence a priori de l'échange économique. Face: empreinte de la souveraineté politique qui, par la présence du sceau, oblige celui qui donne ou reçoit l'objet à en faire un usage conforme à la volonté royale, base de la fonction libératoire de la monnaie au regard d'une dette. Pile: mise en relation quantitative des pièces les unes avec les autres selon un

\footnotetext{
24 J.-M. Thiveaud et S. Piron, art. cité, p. 288.

25 lbid. , p. 284.

${ }^{26}$ Je tiens à souligner ici que l'anglais a conservé ce sens spécifique de la notion de valeur dans le terme valour qui se distingue de value.

27 J.-M. Thiveaud et S. Piron, art. cité, p. 291-292.
} 
système pondéral, base d'une unité de compte et du prix des choses.

Afin de dégager les caractéristiques complètes de l'institution monétaire, je me suis éloigné de l'analyse de Thiveaud et Piron en faisant intervenir une interprétation de l'émergence de la monnaie structurée selon les trois moments formels du procès d'institutionnalisation que $\mathrm{j}$ 'ai décrit dans mon analyse épistémologique de l'institution. Cette interprétation, je tiens à le souligner, est pour moi une hypothèse explicative, laquelle se fonde sur la théorie des sociétés traditionnelles proposée par Michel Freitag ainsi que sur une relecture des analyses des royautés traditionnelles faites par Aglietta et Orléan dans leur ouvrage La violence de la monnaie et sur une synthèse de travaux historiques sur l'«économie» des sociétés mésopotamiennes ${ }^{28}$.

Dans leur analyse, Thiveaud et Piron font remarquer que le métal servant à la fabrication des pièces de monnaie lydiennes provenait du trésor royal. Les auteurs insistent sur l'importance, pour le roi, de la distribution symbolique de ce trésor à travers le monnayage du lien entre dominants et dominés qui en résulte. Ces pièces de monnaie étaient destinées aux armées royales ${ }^{29}$. Mais les auteurs auraient pu aussi insister sur le rapport entre le trésor royal et la capacité du souverain à mobiliser, par l'impôt, les ressources produites dans son royaume, car le trésor royal n'était pas que le fruit de conquêtes ponctuelles, il était aussi et surtout le fruit du tribut annuel que devaient payer les sujets au roi. Or ce rapport social qu'est le tribut a une caractéristique essentielle: il permet de mettre en équivalence des objets disparates, de faire qu'un objet s'exprime quantitativement dans un autre par la voie de la comparaison, c'est-à-dire de donner un prix aux objets et de les faire exister comme marchandises.

On peut supposer qu'avant la monnaie ces équivalences entre objets étaient circonstancielles, qu'elles résultaient du jugement ponctuel du

\footnotetext{
${ }^{28}$ Les ouvrages suivants m'ont fourni le matériau empirique nécessaire à cette interprétation: J. Bottéro, Mésopotamie, l'écriture, la raison et les dieux, Paris, Gallimard, 1987; J. N. Postgate, Early Mesopotamia: Society and Economy at the Dawn of History, Londres, Routledge, 1992; G. Roux, Ancient Iraq, Londres, Penguin, 1992; P. Wattenmaker, Household and State in Upper Mesopotamia, Washington, Smithsonian, 1998; M. Weber, Économie et société dans l'Antiquité, Paris, La Decouverte, 1999 (1909); M. Weber, Histoire économique, Paris, Gallimard, 1991 (1923).
}

29 J.-M. Thiveaud et S. Piron, art. cité, p. 284. 
souverain ou d'un de ses délégués. L'invention d'une unité de compte en grains ou en barres de métal précieux par les administrations royales en Mésopotamie et en Égypte a permis de fixer les systèmes de mise en équivalence et, finalement, de les utiliser comme outil juridique pour remplacer la loi du talion par des peines compensatoires. Ces systèmes d'unité de compte n'étaient que des instruments de comptabilité et de gestion du tribut utilisés par les fonctionnaires de l'administration royale, et la capacité de mise en équivalence d'objets qui en résultait était limitée puisqu'elle n'existait que dans le rapport immédiat de ces objets au tribut et donc au trésor ${ }^{30}$. La création de la monnaie et sa distribution parmi une partie de la population ont permis d'inverser et de généraliser ce rapport entre le trésor et les objets; la monnaie est ainsi devenue véritablement médiation de la «valeur», puisque la mise en équivalence n'était plus seulement abstraite - rapport d'une chose à une unité de compte - mais réelle et matérielle: la chose ne fait pas que s'exprimer en une quantité déterminée, elle s'échange contre cette quantité, elle se transforme dans cette quantité, et ce sans effectuer le détour par le trésor royal en passant par le tribut (ou le tribunal dans le cas d'une peine compensatoire monétarisée). Le pouvoir d'aliénation du trésor royal ou du tribunal au regard de la normativité culturelle devient dès lors une référence proto-idéologique extérieure qui légitimise et structure les nouvelles pratiques monétaires interindividuelles, par exemple en fixant la «valeur» des choses selon une norme du juste prix. La monnaie peut ainsi émerger dans un espace exempt de rapports marchands et faire exister un ensemble d'objets en tant que valeur d'échange, mais d'abord dans l'échange entre la couronne et la société. Cette réification des objets en valeur d'échange, n'en déplaise aux économistes, n'est pas fixée par les canons de «loi de l'offre et de la demande», mais par la norme du juste prix coutumier de l'objet.

Si la monnaie naît en Lydie, c'est dans les cités grecques qu'elle s'enracinera, entre le vie et le ve siècle av. J.-C., comme institution sociale. C'est de là qu'elle se diffusera, quoique plus tardivement, dans les autres sociétés traditionnelles de l'Antiquité; la monnaie ne pénétrera les sociétés du Proche-Orient et d'Égypte qu'à la suite des conquêtes d'Alexandre le Grand. Comment expliquer que la Grèce adopte si tôt la

\footnotetext{
${ }^{30}$ Voir, à ce sujet, M. Aglietta et A. Orléan, La violence de la monnaie, ouvr. cité, p. 153-154.
} 
monnaie? Notons, premièrement, que la monnaie, avant d'être médiation des rapports aux choses, ainsi que je l'ai expliqué ci-dessus, est médiation des rapports sociaux entre les individus. Elle s'est diffusée dans la société grecque principalement comme une médiation spécifique des obligations interindividuelles distinctes du don, du service et de l'esclavage. Sa caractéristique particulière dans le contexte de ces institutions des sociétés traditionnelles est sa puissance libératoire s'agissant d'une dette. La circulation de la monnaie, contrairement à la circulation des dons, n'est pas cyclique mais linéaire: chaque prestation monétaire n'en appelle pas une autre, la monnaie ne tisse pas des obligations, elle les éteint ${ }^{31}$. Elle tient ce pouvoir libératoire de sa participation au principe de souveraineté politique. La participation de la monnaie à la souveraineté libère en quelque sorte cette dernière de la trame des obligations coutumières (des usus) qui, normalement, enserre les rapports interindividuels; la monnaie porte en elle la potentialité de l'arbitraire, de l'abusus réservé au souverain. En distribuant la monnaie, le souverain diffuse sa souveraineté dans la société, car il permet aux individus de s'engager de manière autonome les uns vis-à-vis des autres, libérés des normes traditionnelles culturelles-symboliques, ainsi qu'en l'absence de la personne du souverain. Ces rapports monétarisés s'implantent dans les sociétés grecques pendant la période d'instabilité politique du régime des tyrans, avant la période classique. La renaissance de la monnaie à l'extérieur de la Lydie s'expliquerait par le fait que la monnaie permettait de fonder symboliquement le pouvoir d'un souverain usurpateur ne pouvant revendiquer a priori la légitimité divine de son pouvoir ${ }^{32}$.

L'institution monétaire est avant tout un produit politique, et c'est dans la sphère du rapport à la souveraineté qu'elle intervient dans les sociétés traditionnelles de l'Antiquitế, avant de médiatiser des rapports d'échange qui ne deviendront des rapports économiques qu'après un séjour dans les interstices de la société médiévale. Avant d'être instrument de circulation dans l'espace privé «horizontal» de l'échange économique, la monnaie a été instituée comme médiation des dettes entre les sujets à travers leur dette symbolique commune vis-à-vis du souverain. La monnaie n'est pas seulement exogène à la sphère de l'économie, elle précède l'émergence de

\footnotetext{
31 Voir, à ce sujet, les travaux d'Aldo Haesler sur la monnaie, plus particulièrement son analyse de la différence entre échange symbolique et échange économique (A. Haesler, «Genèse et dissolution de l'économique», Société, no 3, 1988, p. 27-62).

32 J.-M. Thiveaud et S. Piron, art. cité, p. 274.
} 
cette forme de pratique, et elle a été une condition nécessaire à son développement.

Il ressort de mon interprétation que les fonctions remplies par la monnaie dans un système économique dérivent des caractéristiques de la monnaie telles qu'elles sont objectivées et matérialisées dans sa forme. Celles-ci sont le reflet du procès d'institutionnalisation de cette médiation dans les sociétés traditionnelles; elles se présentent dans la structure pile/face qui renvoie aux deux formes de souveraineté qui s'objectivent dans la monnaie. Face: la puissance libératoire au regard des rapports intersubjectifs; pile: la puissance libératoire au regards des rapports aux choses. Par ces deux caractéristiques, la diffusion de la monnaie fonde un espace d'autonomie à l'intérieur de la société, entre la pure domination politique et la trame des obligations coutumières, espace que l'on pourrait qualifier de civil dans la mesure où il contient les structures nécessaires (mais non pas suffisantes) au développement des institutions typiques de la société civile bourgeoise: contrat et propriété.

Le vieil adage selon lequel la monnaie serait «la liberté frappée» prend ici tout son sens. Mais il ne faut pas oublier que la frappe, pour être visible, doit se faire dans une substance. Cette substance de la monnaie, l'alliage de métaux précieux provenant du trésor royal, est ce qui conserve la relation entre la monnaie et son origine: la souveraineté politique. La puissance symbolique de la monnaie, son double pouvoir libératoire envers les gens (les statuts et les rôles) et envers les choses (les usages) dépend finalement de ce rapport au pouvoir politique, car c'est le souverain qui possède le secret de la valeur de la monnaie et c'est en tant que "valeur» que la potentialité libératoire de la monnaie s'actualise ${ }^{33}$. Les deux qualités institutionnelles de la monnaie ne peuvent médiatiser des rapports sociaux que dans la mesure où elle se transforme en une «quantité ${ }^{34} »$, une valeur

\footnotetext{
33 Il se pourrait bien que ce soit cette différence de nature entre les caractéristiques de la monnaie qui ait permis aux économistes de nier pendant très longtemps l'existence d'une troisième fonction de la monnaie, à côté de ses fonctions d'instrument d'échange et d'étalon de valeur, soit celle-là même qui concerne sa substance, la monnaie comme réserve de valeur.

${ }^{34}$ Le double pouvoir libératoire de l'institution monétaire représente ses caractéristiques formelles, dans le sens que celles-ci produisent et régissent une forme d'interaction. Par contre, la substance de la monnaie, la valeur, est la substance (le contenu) même dans laquelle doivent se dissoudre les rapports ainsi «libérés», ceux-ci doivent littéralement devenir «quantités». La formule de Marx, M-A-M, exprime bien cela, je crois.
} 
d'échange, et cette transformation est relative, car elle dépend d'un acte contingent de souveraineté politique, c'est-à-dire d'une volonté qui doit légitimer ses gestes. Le fondement en dernière instance de la valeur de la monnaie est ainsi un rapport fiduciaire, et rapidement la valeur de la monnaie va dépendre en partie du prix que le souverain est prêt à payer pour les éléments de la substance monétaire, c'est-à-dire le prix de l'or et de l'argent ${ }^{35}$.

Avant même l'émergence des économies modernes, la souveraineté diffuse de la monnaie telle qu'elle s'exprime dans la double fonction formelle de l'institution monétaire a pu s'ériger contre la souveraineté originale de l'acte de création monétaire et limiter l'arbitraire du pouvoir politique. Le pouvoir politique a pu conserver sa souveraineté sur la monnaie seulement dans la mesure où il pouvait limiter à son tour légitimement le champ d'application de la puissance formelle de la monnaie: en limitant le droit de circulation de la monnaie par un taux de change imposé aux monnaies étrangères, en monopolisant les exploitations minières, en obligeant les individus à vendre leur monnaie pour la refonte à un taux forcé au moment d'une nouvelle émission de monnaie. Malgré ces mesures, la tension entre la valeur symbolique de la monnaie et la valeur marchande de sa substance s'enracinera comme contradiction politique fondamentale au sein des sociétés où est présente l'institution monétaire. Car, en dernière analyse, la valeur de la monnaie, sa valeur d'échange, est devenue un rapport fiduciaire entre deux ordres de souveraineté, la souveraineté politique et celle des sujets de l'espace civil. Ce que nous appelons régulation monétaire, c'est l'ensemble des pratiques politiques qui relèvent de ce rapport fiduciaire entre l'État et la valeur de la monnaie, et les différents modes de régulation monétaire (que je vais maintenant analyser) sont autant d'arrangements institutionnels en vue de surmonter la contradiction de la valeur de la monnaie. Quand je parle de régulation monétaire, je fais référence à la régulation de cette troisième

\footnotetext{
35 Ce qui ne veut pas dire que la valeur de la monnaie corresponde à la valeur marchande de sa substance (l'or, l'argent, le cuivre) qui existerait en elle-même et pour elle-même. $\grave{A}$ ce sujet, Aglietta et Orléan remarquent, dans le cas des cités grecques: «Les comparaisons effectuées par les numismates ont montré que la déconnexion de la valeur instituée des monnaies frappées par rapport aux équivalences établies entre métaux non frappés s'est produite dès le début du monnayage. Plus fondamentalement c'est la valeur du métal qui s'est fixée par référence à la monnaie approuvée par le pouvoir politique.» (M. Aglietta et A. Orléan, La violence de la monnaie, ouvr. cité, p. 160.).
} 
dimension de l'institution monétaire. Le tableau 2 présente de manière synthétique les caractéristiques sociologiques de l'institution monétaire telles que je viens de les dégager.

Tableau 2

Caractéristiques sociologiques de l'institution monétaire

\begin{tabular}{|l|l|l|l|l|l|}
\hline $\begin{array}{l}\text { Moment de } \\
\text { la forme } \\
\text { monétaire }\end{array}$ & $\begin{array}{l}\text { Caractéristi- } \\
\text { ques insti- } \\
\text { tutionnelles }\end{array}$ & $\begin{array}{l}\text { Fonction } \\
\text { sociale }\end{array}$ & $\begin{array}{l}\text { Rapport au } \\
\text { politique }\end{array}$ & $\begin{array}{l}\text { Type de } \\
\text { régula- } \\
\text { tion }\end{array}$ & $\begin{array}{l}\text { Réification } \\
\text { économique } \\
\text { des fonctions } \\
\text { sociales (cf. } \\
\text { tableau 1) }\end{array}$ \\
\hline $\begin{array}{l}\text { Pile: marque } \\
\text { du système } \\
\text { royal de } \\
\text { mesures } \\
\text { (poids) }\end{array}$ & $\begin{array}{l}\text { Inscription } \\
\text { dans un } \\
\text { système } \\
\text { d'équivalen- } \\
\text { ces selon un } \\
\text { mode quan- } \\
\text { titatif }\end{array}$ & $\begin{array}{l}\text { Pouvoir } \\
\text { libératoire à } \\
\text { l'endroit des } \\
\text { choses }\end{array}$ & $\begin{array}{l}\text { Indirect, } \\
\text { régulation } \\
\text { juridique du } \\
\text { droit de } \\
\text { propriété }\end{array}$ & $\begin{array}{l}\text { Formelle } \\
\text { universel }\end{array}$ \\
\hline $\begin{array}{l}\text { Face : sceau } \\
\text { royal }\end{array}$ & $\begin{array}{l}\text { Diffusion de } \\
\text { la souve- } \\
\text { raineté de } \\
\text { l'autorité } \\
\text { politique } \\
\text { dans la pièce }\end{array}$ & $\begin{array}{l}\text { Pouvoir } \\
\text { libératoire } \\
\text { vis-à-vis des } \\
\text { gens }\end{array}$ & $\begin{array}{l}\text { Indirect, } \\
\text { régulation } \\
\text { juridique des } \\
\text { contrats }\end{array}$ & Formelle & $\begin{array}{l}\text { Caractère } \\
\text { libératoire }\end{array}$ \\
\hline $\begin{array}{l}\text { Substance } \\
\text { métallique, } \\
\text { alliage de } \\
\text { métaux } \\
\text { précieux du } \\
\text { trésor royal }\end{array}$ & $\begin{array}{l}\text { Expression } \\
\text { de la valeur } \\
\text { comme mode } \\
\text { d'être social } \\
\text { particulier }\end{array}$ & $\begin{array}{l}\text { Reproduction } \\
\text { de la valeur } \\
\text { par la trans- } \\
\text { mutation } \\
\text { d'usages* en } \\
\text { valeurs } \\
\text { échangeables }\end{array}$ & $\begin{array}{l}\text { Direct, } \\
\text { monopole } \\
\text { politique du } \\
\text { monnayage }\end{array}$ & Politique & $\begin{array}{l}\text { Réserve de } \\
\text { valeur }\end{array}$ \\
\hline
\end{tabular}

'La notion d'usage renvoie tant à des objets matériels, des choses, qu'à des relations sociales particulières telles que le service pour autrui ou le droit à l'alliance matrimoniale (la dot).

\section{Le politique et la monnaie: vers une typologie des formes de régulation monétaire}

Dans les sociétés traditionnelles, le rapport entre l'État et l'institution monétaire est immédiat, c'est un rapport que l'on peut qualifier de simple: l'État est titulaire du droit de battre monnaie, il détermine la valeur métallique de la monnaie en spécifiant le contenu en métaux précieux des pièces et bat lui-même ou délègue ce droit à autrui. L'autonomie tant de la 
monnaie que de l'activité marchande est faible, en ce sens que la légitimité de ces pratiques reste sous la dépendance immédiate d'une sanction politique. La monnaie dans ces sociétés est «matérielle», elle n'existe pas en dehors de sa forme monnayée, la «masse monétaire» correspond au nombre de pièces monnayées par l'autorité politique.

La régulation monétaire dans un rapport simple État-monnaie concerne très directement la nature de ces pièces de monnaie. Cela se traduit soit par le contrôle de la qualité des pièces de monnaie - la capacité de faire refondre des pièces usées pour que la monnaie conserve symboliquement sa valeur -, soit par la décision de produire de nouvelles pièces d'une valeur inférieure ou supérieure à une monnaie «de compte» déjà existante. Cette forme de régulation immédiate de la monnaie implique par le fait même l'inexistence d'un système financier élaboré comme médiation du rapport des sujets à la monnaie. C'est la raison principale pour laquelle les pouvoirs politiques de l'Europe médiévale ne pourront encadrer et contrôler les réseaux financiers, en pleine croissance et créés par les commerçants d'argent à la fin du Moyen Âge, par le biais de cette forme de régulation politique. Ces réseaux se sont appuyés sur la régulation juridique des dimensions formelles de l'institution monétaire pour légitimer une forme particulière de pratique monétaire qui vient interroger le rapport du pouvoir politique à la troisième dimension de l'institution monétaire, celle qui a trait à la valeur de la monnaie comme expression de la puissance du trésor royal.

Les commerçants de l'argent ont pu contester le monopole public de la régulation monétaire au profit d'une forme de régulation privée basée sur leur propre capacité d'émettre de nouvelles formes de monnaie à partir de leur trésor privé. C'est à l'intérieur de ces circuits monétaires privatisés qu'apparaîtra la forme de monnaie qui sera dominante dans la modernité, le billet et son complément, la monnaie scripturale. Leur apparition marque le passage d'une création monétaire entièrement étatisée, la frappe de pièces, à une création privatisée à l'intérieur des systèmes financiers. Pendant une grande partie de la modernité, la monnaie a pris la forme de «billets de banque» ou d'inscriptions dans les livres de comptes, c'est-à-dire de monnaie émise par des institutions financières privées dont la valeur renvoie à celle de la monnaie métallique étatique. Or cette référence était «idéale» en ce sens que le rapport entre la monnaie privée et la monnaie métallique reposait sur la qualité de l'institution émettrice. Le rapport fiduciaire entre la monnaie et les sujets du politique (la 
contradiction de la souveraineté monétaire) s'est complexifié, puisqu'au rapport fiduciaire politique s'est ajouté un nouveau rapport similaire entre l'institution financière émettrice de monnaie et les «sujets économiques».

Expression de l'accumulation de trésors privés, la substance de cette nouvelle monnaie n'est plus l'or ou l'argent issu du trésor royal; elle repose plutôt sur le dynamisme même de l'activité marchande, c'est-à-dire que la substance des nouvelles formes monétaires est devenue le capital. Cette création monétaire privée était liée à la capacité de faire circuler et fructifier les dettes et les créances marchandes. «Ainsi le transfert de dette a-t-il pour effet de transformer le signe monétaire dans la circulation en signature privée $e^{36} . »$ Cela marque le passage d'une monnaie servant d'intermédiaire aux échanges et dont la valeur repose sur sa capacité de médiation entre des objets "valeur d'usage» qualitativement étrangers (selon la formule de Marx, M-A-M ) à une monnaie dont la valeur est médiatisée par le processus de valorisation du capital, de transformation productive de valeur d'usage en valeur d'échange (A-M-A'). Dans le premier cas, la monnaie se réalise dans sa capacité libératoire; dans le deuxième cas, elle se réalise dans l'accumulation et surtout dans sa capacité de mobiliser le travail par la généralisation du salariat comme forme de rapport d'une classe d'individus à la monnaie ${ }^{37}$. Cette nouvelle monnaie-capital est contemporaine de la naissance du capitalisme comme forme dominante des rapports sociaux. Elle implique la transformation du «commerce d'argent» de la fin du Moyen Âge en capital financier qui se matérialise en un système financier composé d'un ensemble d'institutions économiques telles que les Bourses, les «places financières» et surtout les banques.

Le rapport de l'État à la monnaie, comme celui de l'ensemble des sujets économiques, est dès lors médiatisé par ces institutions financières privées. La dimension fiduciaire de l'institution monétaire s'en trouve radicalement transformée, puisque la valeur de la monnaie, lieu de contradiction potentielle entre deux ordres de souveraineté, repose maintenant sur la puissance privée plutôt que publique. La réaffirmation de l'emprise du politique sur l'institution monétaire par le développement d'une nouvelle forme de régulation monétaire devra maintenant se faire par

36 M. Aglietta et A. Orléan, La violence de la monnaie, ouvr. cité, p. 164.

${ }^{37}$ Ibid., p. 177. 
le biais d'une emprise de l'État sur ces institutions et appareils du système financier. Ce processus ne pouvait se réaliser dans le cadre des formes traditionnelles de souveraineté politique; il est contemporain de la transformation du rapport de l'État avec la société en général qui a caractérisé le passage des sociétés traditionnelles aux sociétés modernes. Il s'est concrétisé par la création des banques centrales comme appareils publics de régulation institutionnelle des institutions financières privées.

Une typologie sociologique de la régulation monétaire peut dès lors différencier des formes de régulation monétaire en fonction de la forme du rapport de l'État avec la monnaie qui varie selon les types de société: forme immédiate «autoritaire» dans le cas de la régulation institutionnelle propre aux sociétés traditionnelles, impliquant une institution monétaire émanant d'une autorité politique dont la légitimité est assurée par une référence à une transcendance extra-mondaine; forme médiate «institutionnelle et politique» dans le cas du mode de régulation propre aux sociétés modernes, impliquant une institution monétaire devenue institution économique. Chacune de ces formes de régulation institutionnelle comporte des variantes historiques et civilisationnelles spécifiques. On pourrait, par exemple, distinguer, dans la forme immédiate traditionnelle, la régulation seigneuriale typique de l'époque féodale d'une régulation «impériale» dans l'Empire romain et d'une régulation «tyrannique» propre aux cités-États de la Grèce. La forme de régulation médiate «politico-institutionnelle» propre aux sociétés modernes se différencie pour sa part selon l'orientation "normative» du rapport qui existe entre l'État et la monnaie qui est inséparable du rapport plus global de l'État avec l'économie et la société, institué grâce au discours de l'économie politique au début et tout au long de la modernité.

La réaffirmation de la souveraineté politique sur la monnaie à la suite de l'épuisement de la régulation seigneuriale a pris la forme d'un discours idéologique de légitimation de la régulation politique qui reconnaissait $a$ priori l'autonomie ${ }^{38}$ des pratiques économiques et, donc, des «sujets de la société civile» et qui redéfinissait, sur une base séculaire et rationaliste, la forme de la régulation de cette sphère en lui assignant comme rôle d'assurer les meilleures conditions non seulement de reproduction de ses

\footnotetext{
38 Voir, à ce sujet, G. Bourque et J. Duchastel, Restons traditionnels et progressifs, Montréal, Boréal, 1988.
} 
activités, mais aussi de leur développement, au nom d'une représentation particulière de l'intérêt général. Cette irruption du moment idéologique, sous la forme d'une idéologie politique, dans la régulation monétaire est centrale dans la compréhension du rapport de l'État à la monnaie dans la modernité et renvoie plus globalement à la nouvelle forme de légitimation du pouvoir propre aux sociétés modernes: le discours politique. La régulation politique moderne ne repose plus sur la décision arbitraire du souverain de droit divin, elle doit s'orienter selon un ensemble de principes légitimes visant une finalité discutée et discutable dans la sphère publique. Ces principes n'appartiennent pas uniquement à la sphère de la régulation monétaire, ils structurent l'ensemble de la régulation économique et se rapportent plus globalement aux principes idéologiques qui légitiment et orientent la régulation politique des rapports sociaux par l'État moderne. Ils se cristallisent dans la forme d'un discours idéologique qui assigne explicitement une ou plusieurs finalités à la régulation politique et qui distingue ces finalités du travail immédiat de régulation des pratiques institutionnelles.

La création des banques centrales et la régulation monétaire moderne

Les banques centrales ont existé dans un premier temps non pas tant comme véhicules d'une politique monétaire, mais comme dépositaires du rapport fiduciaire entre l'État et la monnaie, par leur travail de régulation des fonctions monétaires privatisées. Leurs fonctions étaient celles-là mêmes qui s'étaient unifiées à l'intérieur des systèmes financiers grâce aux pratiques du capital financier, pratiques reposant sur la production de nouvelles formes monétaires à partir des caractéristiques institutionnelles de la monnaie. Les banques centrales exercent leurs fonctions uniquement dans l'espace des rapports entre l'État et le système financier. C'est-à-dire que les fonctions d'une banque centrale touchent à la régulation publique des fonctions de l'institution monétaire par la médiation des rapports entre l'État et le système financier. La mise en place de ces institutions financières publiques marque en même temps une reconnaissance politique de l'autonomie institutionnelle du système financier - une reconnaissance de son appropriation privée de la capacité publique de création monétaire - par le biais de son assujettissement à une instance de régulation de deuxième degré de ces pratiques institutionnelles privées. Le passage d'une forme de régulation monétaire immédiate à une forme de régulation 
médiate politico-institutionnelle par la création des banques centrales est ainsi un moment du passage des sociétés traditionnelles aux sociétés modernes caractérisées par l'«institutionnalisation de la capacité d'institutionnalisation».

Afin d'effectuer leur travail de médiation politico-institutionnel entre le pôle étatique et le pôle financier, les banques centrales instaureront des régulations selon deux axes distincts, un axe ascendant qui va de l'économique au politique et un axe descendant qui va du politique à l'économique. Ces régulations se déploieront selon la logique institutionnelle des trois fonctions monétaires. Le rapport de l'État à la banque centrale est double: un rapport strictement économique, l'État est client de la banque (axe ascendant); un rapport politique, la banque est mandatée par l'État pour veiller à la bonne marche du système financier et à la protection de la valeur de la monnaie nationale (axe descendant). Le rapport entre la banque centrale et les institutions du système financier est aussi double: d'une part, les institutions financières sont clientes de la banque centrale, tout comme celle-ci est un acteur économique «comme les autres» dans le marché (axe ascendant); d'autre part, la banque supervise les activités de ces institutions et protège l'intégrité du système financier (axe descendant). Le tableau qui suit résume ces différentes dimensions de la régulation monétaire moderne.

On peut, à partir du tableau 3, différencier et hiérarchiser le travail de régulation monétaire effectué par les banques centrales: le travail associé à l'axe ascendant (de l'économique au politique) correspond à des opérations économiques et le travail associé à l'axe descendant (du politique à l'économique) prend la forme d'un discours de régulation politique. Ce discours constitue l'a priori des opérations économiques faites par les banques centrales, c'est-à-dire qu'il est le moment de légitimation de la régulation monétaire moderne. Les opérations économiques liées à la régulation monétaire sont les outils permettant l'emprise effective des banques centrales sur les systèmes financiers, tandis que le discours de la régulation monétaire détermine et oriente ce travail de régulation en lui assignant des finalités légitimes, le moment discursif étant le véritable contenu de la régulation monétaire. Ce moment discursif se déploie à l'intérieur de l'espace plus global du discours de régulation politique de l'économie. 


\section{Tableau 3}

\section{Fonctions monétaires}

\section{et fondements de la régulation monétaire moderne}

\begin{tabular}{|c|c|c|c|c|}
\hline $\begin{array}{l}\text { Dimensions de } \\
\text { l'institution } \\
\text { monétaire } \\
\text { moderne }\end{array}$ & \multicolumn{2}{|c|}{$\begin{array}{l}\text { Rapport banque-système } \\
\text { financier; pratiques de } \\
\text { régulation monétaire du système } \\
\text { financier }\end{array}$} & \multicolumn{2}{|c|}{$\begin{array}{l}\text { Rapport État-banque centrale; } \\
\text { pratiques de régulation } \\
\text { monétaire de la banque centrale } \\
\text { par l'État }\end{array}$} \\
\hline $\begin{array}{l}\text { Fonctions } \\
\text { monétaires }\end{array}$ & Axe ascendant & Axe descendant & Axe ascendant & Axe descendant \\
\hline $\begin{array}{l}\text { Équivalent } \\
\text { universel }\end{array}$ & $\begin{array}{l}\text { Compensation } \\
\text { entre les } \\
\text { comptes des } \\
\text { institutions } \\
\text { financières } \\
\text { nationales }\end{array}$ & $\begin{array}{l}\text { Régulation des } \\
\text { formes } \\
\text { acceptables de } \\
\text { moyens de } \\
\text { paiement et de } \\
\text { la solvabilité des } \\
\text { institutions } \\
\text { financières par } \\
\text { la détermina- } \\
\text { tion des réser- } \\
\text { ves obligatoires }\end{array}$ & $\begin{array}{l}\text { Gestion des } \\
\text { réserves de } \\
\text { l'État }\end{array}$ & $\begin{array}{l}\text { Détermination } \\
\text { de la structure } \\
\text { du système } \\
\text { financier }\end{array}$ \\
\hline $\begin{array}{l}\text { Caractère } \\
\text { libératoire (n'est } \\
\text { garanti qu'à } \\
\text { l'intérieur d'un } \\
\text { territoire } \\
\text { politique) }\end{array}$ & $\begin{array}{l}\text { Change de } \\
\text { devises } \\
\text { étrangères pour } \\
\text { les institutions } \\
\text { financières } \\
\text { nationales }\end{array}$ & $\begin{array}{l}\text { Régulation de la } \\
\text { valeur externe } \\
\text { de la monnaie } \\
\text { par un contrôle } \\
\text { du taux de } \\
\text { change }\end{array}$ & $\begin{array}{l}\text { Gestion du } \\
\text { compte des } \\
\text { réserves de } \\
\text { monnaies } \\
\text { étrangères }\end{array}$ & $\begin{array}{l}\text { Détermination } \\
\text { du taux de } \\
\text { change } \\
\text { souhaitable }\end{array}$ \\
\hline $\begin{array}{l}\text { Réserve de } \\
\text { valeur }\end{array}$ & $\begin{array}{l}\text { Créancier en } \\
\text { dernier recours } \\
\text { pour les } \\
\text { institutions } \\
\text { financières } \\
\text { nationales }\end{array}$ & $\begin{array}{l}\text { Régulation de la } \\
\text { valeur interne de } \\
\text { la monnaie par } \\
\text { un contrôle du } \\
\text { taux d'intérêt }\end{array}$ & $\begin{array}{l}\text { Gestion des } \\
\text { paiements de } \\
\text { l'État }\end{array}$ & $\begin{array}{l}\text { Détermination } \\
\text { des taux } \\
\text { d'interêt } \\
\text { souhaitables }\end{array}$ \\
\hline
\end{tabular}

Comme je l'ai mentionné précédemment, la régulation monétaire moderne se différencie selon la forme de l'État moderne, la régulation monétaire s'inscrit dans l'univers d'un discours de régulation politique de l'économie qui oriente et structure de manière principielle le rapport général de l'État moderne avec la sphère institutionnelle économique. Je ne m'attarderai pas ici aux formes libérale et providentialiste de la régulation monétaire. Je me limiterai à rappeler que ces types d'orientation de la régulation, qui correspondent à deux formes successives de l'État, 
participent de la même structure de médiation politique des pratiques monétaires impliquant l'emprise d'une banque centrale sur les systèmes financiers nationaux et son monopole du contrôle de l'émission monétaire $^{39}$. Donc, il y a non seulement assujettissement de la finance privée à la finance publique (assujettissement formel dans le cas de l'État libéral et matériel ou réel dans le cas de l'État-providence), mais aussi subsomption de l'institution monétaire à l'institution financière publique.

Il est important de souligner, finalement, la profonde politisation de la régulation monétaire moderne, libérale et providentialiste, politisation qui reflète des positions de classe antagoniques fondamentales dans chaque forme d'État. Par exemple, aux États-Unis, le débat sur le bimétallisme et le monométallisme au XIXe siècle, immortalisé dans le conte Le magicien $d^{\prime} \mathrm{O}^{40}$, était typique d'une régulation libérale traversée par un ensemble de conflits entre une classe dominante de capitalistes rentiers et de financiers partisans d'un ancrage de la création monétaire à une marchandise rare (or) et une classe émergente d'entrepreneurs, d'industriels et d'agriculteurs capitalistes partisans d'un ancrage à une marchandise abondante (argent).

Quant à la régulation monétaire providentialiste, elle était fondée sur la nécessité d'un arbitrage entre des intérêts reconnus explicitement comme structurellement antagoniques, c'est-à-dire reflétant le compromis social entre classes à la base de cette forme de l'État. Citons, à titre d'exemple, les finalités constitutives de la Banque du Canada telles que les délimite le préambule de la loi sur l'incorporation de la Banque du Canada:

Considérant qu'il est opportun d'établir une banque centrale au Canada pour régler le crédit et la monnaie dans le meilleur intérêt de la vie économique de la nation, pour contrôler et protéger la valeur extérieure de l'étalon monétaire national et pour mitiger, par son influence, les fluctuations du niveau général de la production, du commerce, des prix et de l'emploi de la main-d'œuvre, autant que possible dans le cadre de

39) Ce qui n'empêche pas une délégation de ce monopole à des institutions privées.

4) L'auteur du conte, L. Frank Baum, était un partisan du bimétallisme. L'ensemble du conte est une allégorie dénonçant les effets du monométallisme. Le magicien d'Oz représente les financiers de Boston et de New York, les compagnons de Dorothée, les classes soumises aux financiers. Dorothée rencontre le magicien d'Oz au bout de la route pavée d'or et elle retrouve sa maison prospère, sa famille et une vie heureuse pour toujours grâce à ses souliers d'argent. 
l'action monétaire, et généralement de favoriser la prospérité économique et financière du Dominion ${ }^{41}$.

On retrouve là les éléments du carré magique keynésien (PIB, inflation, chômage et balance commerciale) qui traduisent eux-mêmes les intérêts économiques antagoniques entre travail et capital, dans le contexte d'une relative «euthanasie» des rentiers ${ }^{42}$, c'est-à-dire d'un dépassement des rapports contradictoires qui structuraient la régulation monétaire libérale par une nouvelle opposition de classe dynamique.

La particularité de la régulation néolibérale est de s'instaurer sur la base d'une absence explicite de référence à une contradiction entre des classes socioéconomiques structurellement opposées les unes aux autres. En effet, la régulation néolibérale se met en place en revendiquant une dépolitisation explicite des rapports monétaires. Plutôt que de reconnaître des oppositions de classe, elle prétend unifier la société en étendant un rapport rentier à la monnaie à l'ensemble des couches sociales. Reconnaissant la diffusion massive de positions rentières, entre autres par l'investissement institutionnalisé ${ }^{43}$, dans l'ensemble de la société, la régulation néolibérale peut ainsi affirmer agir au nom d'un intérêt général privé dont l'universalité peut rivaliser avec l'intérêt général public exprimé dans le compromis providentialiste. De plus, encore en opposition avec le providentialisme, la régulation néolibérale reconnaît l'appartenance des acteurs économiques à une sphère de pratique mondialisée, donc extranationale, et elle ne vise pas la domestication ou le confinement au territoire national de ces acteurs ou de leurs pratiques. La régulation monétaire néolibérale visera donc à soutenir coûte que coûte les revenus financiers par l'adoption, à l'échelle nationale, d'une politique énergique de désinflation en même temps qu'elle encouragera la consolidation de la capacité de régulation privée des marchés financiers mondialisés en arrimant son travail de régulation de la valeur externe de la monnaie (taux de change) aux fluctuations de ces marchés ${ }^{44}$. Dans les deux cas, l'effet

41 Cité dans A. Raynauld, Institutions économiques canadiennes, Montréal, Beauchemin, 1964, p. 220.

${ }^{42}$ En ce sens, le débat ayant entouré la création de la Banque du Canada aurait été l'un des premiers éléments annonçant la transition de l'État libéral à l'État-providence au Canada.

${ }^{43}$ Régime enregistré d'épargne-retraite (REER), fonds mutuels, assurances, etc.

44 Pour une analyse plus complète, on peut consulter $\mathrm{R}$. Boyer, Le politique à l'ère de la mondialisation et de la finance: le point sur quelques recherches régulationnistes, Paris, 
premier de cette orientation de la régulation monétaire sera de démanteler et de court-circuiter les mécanismes de régulation économique typiques de la période providentialiste, tels que le contrôle des marchés de change, la domestication des portefeuilles financiers, les politiques budgétaires et fiscales contre-cycliques, etc.

La contradiction structurante dans cette forme de régulation sera celle qui oppose directement le pouvoir politique et la régulation publique à la puissance financière privée et mondialisée couplée à sa capacité de régulation marchande privée. Ainsi s'inverse, dans le cadre de la régulation néolibérale, cette donne fondamentale de la régulation monétaire moderne, l'assujettissement de la finance privée à la finance publique. L'extrait suivant d'un discours charnière du gouverneur de la Banque du Canada, Gordon Thiessen, prononcé en 1996, résume de façon éloquente cette reconfiguration de la régulation monétaire dans le cadre de l'État néolibéral:

[La] Banque du Canada ne peut pas fixer arbitrairement les taux d'intérêt au niveau qui lui convient. Ce sont principalement les épargnants, les investisseurs et les emprunteurs - à la fois canadiens et étrangers - qui déterminent le niveau des taux d'intérêt en fonction de ce qu'ils considèrent comme un rendement juste sur les marchés financiers. [...] Pour que notre situation financière soutienne les progrès de l'économie, il est crucial que les gouvernements s'engagent de façon crédible à ramener l'état de leurs finances sur une trajectoire viable et que la Banque reste inébranlable dans son engagement envers la stabilité des prix ${ }^{45}$.

La régulation monétaire néolibérale reflète ainsi, tout en y contribuant, la production des transformations contemporaines de la forme de l'institution monétaire que j'ai évoquée au début de l'article, son déplacement de la sphère de la finance publique vers celle de la finance privée. Je ne peux, dans l'espace qui reste, me pencher sur l'ensemble de ce procès de transformation de la régulation et de l'institution monétaire. Je me contenterai, pour terminer, de faire une brève présentation critique des postulats théoriques sous-jacents à la régulation monétaire néolibérale.

CEPREMAP, cahier no 9820, 1998.

${ }^{45} \mathrm{G}$. Thiessen, «Les orientations et interactions de la politique monétaire et de la politique budgétaire», allocution prononcée le 19 janvier 1996, Ottawa, Banque du Canada, p. 7. 
Aperçu de la régulation monétaire néolibérale: dissolution de l'institution monétaire et dépolitisation de la régulation des pratiques monétaires

La régulation monétaire néolibérale va se donner une conception de la monnaie, du rôle d'une banque centrale et du rapport entre le politique et la monnaie et l'économie en général en s'inspirant des postulats de la théorie économique "monétariste» dont le principal instigateur est Milton Friedman. Je n'ai pas l'intention de faire une présentation critique exhaustive de la doctrine et de la théorie de cet économiste. Je veux simplement dégager les éléments qui ont fait du monétarisme le référent «épistémique» de la régulation monétaire néolibérale.

Le monétarisme avait comme point de départ une critique des limites et des paradoxes des anciennes politiques économiques keynésiennes, critique dont le fondement est la croyance en une adhésion à la stabilité spontanée du marché, du moins dans le moyen terme. Les monétaristes, qui renaturalisent ainsi le rôle économique de l'État et réinventent la vision libérale du marché autorégulateur, sont de fervents défenseurs de la nonintervention et de la déréglementation, sauf en ce qui concerne la politique monétaire ${ }^{46}$.

Pourquoi limiter l'interventionnisme à la régulation monétaire? Parce que les mécanismes classiques de la politique économique keynésienne n'ont pas d'emprise positive sur les cycles économiques et le développement d'une économie en général, étant donné que les finalités de la régulation économique providentialiste - plein emploi, croissance, stabilité des prix et équilibre extérieur - sont toutes, à moyen terme, sauf dans le cas de la valeur de la monnaie, déterminées par les équilibres spontanés des mécanismes du marché. Ce sont des phénomènes endogènes au fonctionnement de l'économie de marché, tandis que les phénomènes monétaires sont en partie exogènes.

L'argument anti-interventionniste des monétaristes se fonde sur le postulat de la structure dichotomique des systèmes économiques: les distorsions résultant de l'intervention politique dans l'économie se manifestent sous la forme d'un décalage entre le dynamisme naturel de l'économie et le secteur monétaire. Le point culminant de ce décalage est

${ }^{46} \mathrm{M}$. Beaud et G. Dostaler, La pensée économique depuis Keynes, édition abrégée, Paris, Seuil, 1995, p. 178-179. 
Ia contradiction entre la demande de monnaie et l'offre de monnaie, la première étant régie par la logique économique, tandis que la seconde est assujettie à la volonté politique. Pour Friedman:

[...] l'offre de la monnaie, déterminée par les autorités monétaires, est beaucoup plus volatile que la demande, qui découle des comportements des agents. Il s'ensuit que les changements de la valeur de la monnaie, donc du niveau général des prix, sont fondamentalement déterminés par l'offre de monnaie. Les variations de la quantité nominale de monnaie agissent à court terme sur les quantités et l'emploi et à long terme leurs effets sont purement nominaux ${ }^{47}$.

À court terme, une mesure de stimulation économique keynésienne, par injection de liquidités dans l'économie soit sous la forme d'une politique monétaire laxiste, soit sous la forme de dépenses publiques ou de mesures fiscales redistributrices, peut avoir un effet sur le taux de croissance et d'emploi, mais, à long terme, ces gains seront atténués par une diminution de la valeur de la monnaie, c'est-à-dire un ajustement de la valeur nominale de la monnaie par l'inflation. En ce sens, toute mesure interventionniste de stimulation de l'économie se transforme tôt ou tard en inflation, et c'est par ce moyen que se rétablit l'équilibre naturel de l'économie. Le décalage temporel entre l'équilibre nominal et l'équilibre réel s'explique par le phénomène «d'illusion monétaire»: l'incapacité, pour les acteurs économiques dans le court terme, de comprendre l'effet de l'inflation sur la valeur réelle des marchandises (travail, biens ou services) qu'ils offrent.

Les postulats du monétarisme sont donc les suivants. Puisque la régulation macroéconomique, à travers les politiques budgétaires, fiscales et monétaires, n'a d'emprise que sur la sphère monétaire, les seuls véritables problèmes macroéconomiques sont ceux qui touchent la monnaie, c'est-à-dire ceux qui provoquent un décalage entre le dynamisme artificiel de la sphère monétaire et le dynamisme naturel de la sphère réelle. Toute variation des mesures fiscales ou budgétaires, positive ou négative, aura un effet déstabilisateur qui se reflétera dans la sphère monétaire. La sphère réelle, pour sa part, tend par son dynamisme propre vers un état d'équilibre, équilibre qui s'exprime d'ailleurs dans les grandes variables macroéconomiques telles que taux de chômage, variation du PIB, équilibre

47 Ibid., p. 176. 
externe. Le décalage entre la sphère réelle et la sphère monétaire se traduit par le taux d'inflation qui représente un rythme de création monétaire qui ne coïncide pas avec le rythme d'expansion de l'activité économique. Il faut donc restreindre la régulation économique à l'alignement du rythme de création monétaire sur le rythme de croissance de l'économie réelle, ce qui veut dire viser un taux d'inflation zéro, et considérer les variations du taux de croissance, du taux de chômage et de la balance commerciale comme des indicateurs de l'état «naturel» de l'économie compte tenu de ses caractéristiques structurelles. Si l'on veut s'attaquer aux phénomènes du chômage, de la croissance, de l'équilibre externe, on doit s'attaquer aux caractéristiques structurelles de l'économie par le biais de politiques d'ajustement structurel et systémique.

D'un point de vue monétariste, la régulation macroéconomique se réduit alors à intervenir dans la sphère monétaire afin d'ajuster le rythme de création monétaire, pouvoir qui relève de la banque centrale, puisque c'est elle qui contrôle l'offre de monnaie. Ce contrôle du rythme de création monétaire devant de surcroît compenser l'effet naturellement inflationniste des dépenses publiques, la régulation monétaire doit donc encadrer la politique fiscale et la politique budgétaire; c'est de cette manière qu'à l'intérieur de la sphère de la régulation macroéconomique s'inverse le rapport hiérarchique entre régulation monétaire et régulation économique instauré par le providentialisme. De plus, cet assujettissement aux banques centrales des appareils de régulation économique n'est fonctionnel que si cette dernière a conquis son autonomie organisationnelle par rapport au pouvoir politique.

Sur le plan de la régulation monétaire, cela implique finalement une nouvelle hiérarchisation de ses finalités politiques. Plutôt que de viser l'équilibre entre des finalités potentiellement contradictoires reflétant les intérêts antagoniques de différentes classes sociales, le monétarisme érige la stabilité des prix en seule et unique finalité de la régulation monétaire, réduisant les niveaux de la croissance et de l'emploi ainsi que la variation de l'équilibre externe à de simples indicateurs macroéconomiques sur lesquels la régulation n'a d'emprise que par le contrôle de l'inflation, c'està-dire à partir de la sphère monétaire.

L'objet de la régulation monétaire monétariste est ainsi fondamentalement différent de l'objet de la régulation providentialiste, puisqu'il se réduit à un système formel de création et de circulation de signes 
monétaires relativement autonome par rapport aux processus économiques de la sphère réelle qui, dans le contexte providentialiste, sont imbriqués, faut-il le rappeler, dans une structure d'opposition de classes. Le référent de la régulation monétaire néolibérale n'est donc ni le cadre formel des pratiques monétaires de la régulation libérale ni les pratiques et structures du système des institutions financières nationales de la régulation providentialiste; il consiste plutôt dans un système de flux monétaires abstrait dont l'unité est perceptible de deux manières: 1) soit en tant que cons-truction analytique modélisant des pratiques monétaires qui ont été absorbées dans la sphère des pratiques financières privatisées. C'est le sens de l'ensemble des modèles servant à la représentation de la masse monétaire (M1, M2, M3, etc.), qui ont guidé et qui guident encore l'intervention de plusieurs banques centrales; 2) soit sous la forme de la sanction marchande que les acteurs dominants de la finance privée peuvent imposer à l'objet ultime de la régulation monétaire, la valeur de la monnaie dans le cadre de leurs opérations sur les marchés financiers mondialisés. C'est ce que nous rappelle le gouverneur de la Banque du Canada, Gordon Thiessen, quand il affirme que la «Banque du Canada ne peut pas fixer arbitrairement les taux d'intérêt au niveau qui lui convient. Ce sont principalement les épargnants, les investisseurs et les emprunteurs - à la fois canadiens et étrangers - qui déterminent le niveau des taux d'intérêt en fonction de ce qu'ils considèrent comme un rendement juste sur les marchés financiers ${ }^{48}{ }^{\text {». }}$. Ce système de flux monétaires financiarisés constitue le nouvel environne-ment opératoire de la régulation monétaire. Il reflète la double mutation de l'institution monétaire, à savoir son absorption dans des mécanismes finan-ciers privatisés et la dépolitisation de son rapport avec la société par la généralisation d'un rapport privé à la monnaie qui s'impose au rapport public à la monnaie. Il justifie en outre le fait que le contrôle d'une régulation monétaire dépolitisée soit confié à des banques centrales autonomes et à l'abri de l'emprise typique que les instances démocratiques ont sur les appareils publics.

48 G. Thiessen, texte cité, p. 7. 
Pour paraphraser Keynes, la résurrection contemporaine du rentier, dans le cadre de la régulation néolibérale, s'accomplit par l'euthanasie du politique.

\author{
Éric PINEAULT \\ Département de sociologie, \\ Université du Québec à Montréal \\ Groupe en analyse du discours politique (GRADiP)
}

Résumé

Cet article est une contribution au développement d'une théorie institutionnaliste de la monnaie et de la régulation monétaire. Il vise à éclairer le sens des mutations contemporaines des pratiques et politiques monétaires qui sont au cœur du processus de mondialisation du capitalisme, en explorant les racines historiques de la régulation et de l'institution monétaires. Dans une première partie, de nature épistémologique, l'auteur s'emploie, en s'inspirant des travaux de Michel Freitag, à la construction d'une théorie de l'institution monétaire. Cette démarche lui permet de proposer une phénoménologie des pratiques et de la régulation monétaires dans les sociétés traditionnelles et modernes et d'esquisser une typologie des formes de la régulation monétaire. Cette typologie s'inscrit dans la perspective de l'analyse des formes de l'État et de la régulation politique de Bourque et Duchastel. L'article se termine par un examen plus précis de la régulation monétaire néolibérale et laisse entendre que celle-ci participe directement à la légitimation et à la production de l'infrastructure institutionnelle et organisationnelle nécessaire aux mutations actuelles de la monnaie et à l'absorption de l'institution monétaire dans la sphère de la finance privée.

Mots-clés: monnaie, institution monétaire, régulation monétaire, régulation politique, mondialisation financière, régulation économique, forme de l’État, capitalisme, sociologie économique, histoire monétaire.

\title{
Summary
}

This article contributes to the development of an institutionalist theory of money and money regulation. Through a review of the historical roots 
of the institution and regulation of money, it seeks to clarify the meaning of contemporary changes in money practices and policies which are at the heart of capitalism's globalization process. The first section, epistemological in nature and informed by the work of Michel Freitag, is devoted to constructing a theory of the institution of money. It enables the author to propose a phenomenology of money practices and regulation in traditional and modern societies and to sketch a typology of forms of money regulation. This typology is informed by Bourque and Duschastel's analysis of State forms and political regulation. The article concludes with a more detailed examination of neo-liberal money regulation and suggests that this regulation participates directly in the legitmation and production of the institutional and organisational infrastructure necessary to current changes in money and the institution of money in the sphere of private finances.

Key-words: money, institution of money, money regulation, political regulation, financial globalization, economic regulation, State form, capitalism, economic sociology, history of money.

\section{Resumen}

Este artículo es una contribución al desarrollo de una teoría institucionalista de la moneda y de la regulación monetaria. El autor pretende aclarar el sentido de las mutaciones contemporáneas de las prácticas y políticas monetarias que se encuentran en el centro del proceso de mundialización del capitalismo tomando las raíces históricas de la regulación y de la institución monetaria. La primera parte del texto, que tiene un carácter epistemológico, está consacrada a la construcción de una teoría de la institución monetaria que se inspira de los trabajos de Michel Freitag. Este trabajo permite al autor de proponer una fenomenología de las prácticas y de las regulaciones monetarias en las sociedades tradicionales y modernas y de hacer un bosquejo de una tipología de formas de regulación monetaria. Esta tipología se inscribe dentro de la perspectiva de análisis de las formas del Estado y de la regulación política de Bourque y de Duchastel. El artículo concluye con un examen preciso de la regulación monetaria neoliberal, el cual deja entender que ésta participa directamente en la legitimación y en la producción de la infraestructura institucional y organizacional necesaria a las mutaciones actuales de la moneda y a la 
absorción de la institutción monetaria dentro de la esfera de la finanza privada.

Palabras claves: moneda, institución monetaria, regulación monetaria, regulación política, mundialización financiera, regulación económica, forma del Estado, capitalismo, sociología económica, historia monetaria. 